\title{
Advanced electrokinetic characterization of composite
}

\section{porous membranes}

\author{
Anthony Szymczyk", Yonis Ibrahim Dirir, Matthieu Picot, Irène Nicolas, Frédéric Barrière \\ Université de Rennes 1, Institut des Sciences Chimiques de Rennes, UMR CNRS 6226, 263 Avenue du \\ Général Leclerc, 35042 Rennes cedex, France. \\ *Corresponding author, anthony.szymczyk@univ-rennes1.fr
}

\begin{abstract}
The effect of the streaming current flowing through the porous structure of composite membranes during tangential electrokinetic measurements was investigated. It was shown that neglecting this additional path for streaming current may have dramatic implications in the interpretation of the experimental data and on the determination of the membrane zeta potential. Experimental measurements of both streaming current and electrical conductance were performed with two different composite polymer membranes. By following the procedure proposed by Yaroshchuk and Luxbacher (ref. 17 in the present work) it was possible to determine separately the zeta potential of the membrane surfaces and that of their underlying porous structures. This experimental procedure was shown to provide useful information about the efficiency of the functionalization of an ultrafiltration polyethersulfone membrane by positively charged 4-benzyltriphenylphosphonium groups, notably that the chemical modification primarily occurs in the porous substructure of the membrane rather than on its external surface.

Keywords: Electrokinetic characterization; Streaming current; Porous membranes; Membrane functionalization
\end{abstract}




\section{INTRODUCTION}

The zeta potential, defined as the electrostatic potential at the hydrodynamic plane of shear, is a fundamental feature providing useful information about the charge state of a solid surface. Assessing the zeta potential of filtration membranes is particularly attractive because this quantity is correlated with the mechanism of rejection for charged solutes [1]. Moreover, zeta potential is very sensitive to any change in surface electrical properties and it can therefore serve as a probe for various studies in material science dealing with adsorption phenomena, surface ageing, membrane fouling, etc.

In membrane science, a standard method for zeta potential determination consists in the measurement of the so-called transversal (or through-pore) streaming potential which is defined as the pressure-induced electrical potential difference arising between pore ends under zero electrical current condition and no concentration difference across the membrane. Thanks to its experimental simplicity, the measurement of transversal streaming potential has become a standard technique for assessing the electrokinetic properties of porous membranes and it has been largely applied for several decades [2-7].

Although some theoretical approaches have been developed to deal with streaming potential through multilayer membranes [8-11], a meaningful interpretation of data obtained from the transversal technique is difficult with commercial composite membranes for which it is generally not possible to get the underlying support layer(s) separated from the rest of the membrane. To overcome these difficulties the tangential streaming potential technique was applied to the characterization of membranes [12]. In this alternative method, the pressure gradient is no longer applied through the membrane pores but along a channel formed by the skin layers of two identical membranes facing each other with their skin layers. However, several works have pointed out the difficulties associated with the interpretation of tangential streaming potential data for the determination of zeta potential of skin layers of porous membranes [13, 14] because of the additional contribution of the underlying support layers (which act as conducting 
substrates since they are filled with an electrolyte solution during measurements) to the total electric conductance of the system (cell conductance). The contribution of the membrane porous body to the cell conductance was first suggested by Yaroshchuk and Ribitsch [13] and was further proved experimentally by Fievet et al. [14] and Sbaï et al. [15] with ceramic and polymer membranes, respectively.

Alternatively, it has been proposed to measure the streaming current along the skin layer of composite membranes since its interpretation is not complicated either by the surface conductance or by the electrical conduction through the porous sublayers of membranes [16]. However, it has been recently shown that not only the streaming potential but also the streaming current coefficients could be affected by the membrane porous body [17].

In this work, we show that the effect of the additional streaming current flowing through the porous body of composite ultrafiltration membranes has significant implications in the interpretation of experimental data. The additional streaming current flowing through membrane bodies can be viewed as a parasite signal which adds to the streaming current in the channel and makes the interpretation of experimental data more difficult in terms of zeta potential. It is shown, however, that the electrokinetic contribution of porous structures can provide useful information about the functionalization of porous membranes and reveal some interesting details concerning the pattern of membrane modification. This will be illustrated with an ultrafiltration polyethersulfone membrane modified covalently by in situ chemical reduction of an aryldiazonium salt.

\section{THEORETICAL BACKGROUND}

Let us consider an electrolyte solution between two identical charged surfaces facing each other. If a hydrostatic pressure gradient is applied through the channel formed by the two surfaces the liquid is forced to move tangentially to the charged surfaces, pulling the excess of mobile ions within the 
electrical double layers towards the low-pressure side. The electrical current caused by the liquid flow is called the streaming current. If no external circuit is provided as a current loop, the excess of ions within the electrical double layers accumulates at the channel end. This charge build-up gives rise to an electric field that causes a conduction current in the opposite direction of the streaming current. The backconduction current first increases because of gradual charge accumulation at the channel end and it becomes rapidly equal to the streaming current, which prevents any further charge build-up. A steadystate is then achieved and a stable electrical potential difference, called streaming potential, can be measured between the channel ends if an electrometer with a sufficiently high resistance is placed in the external circuit. An alternative technique consists in measuring directly the streaming current, which can be done in absence of electrode polarization by using an electrometer with a low resistance (with respect to the channel resistance) so that the conduction current in the external circuit is almost equal to the streaming current [18].

In the standard electrokinetic theory it is implicitly assumed that the channel through which electrokinetic measurements (streaming current and / or streaming potential) are performed has impermeable walls. In this case, and if the channel height $h_{c h}$ (i.e. the distance between the two surfaces facing each other) is much larger than the Debye length [19], the streaming current $\left(I_{s}\right)$ is linked to the zeta potential $(\zeta)$ by the following well-known relation [20]:

$$
I_{s}=-\frac{W h_{c h} \varepsilon_{0} \varepsilon_{r} \Delta P}{\eta L} \zeta
$$

where $W$ and $L$ are the channel width and length, respectively, $\varepsilon_{0}$ is the vacuum permittivity, $\varepsilon_{r}$ and $\eta$ are the dielectric constant and the dynamic viscosity of the electrolyte solution, respectively, and $\Delta P$ is the pressure difference between channel ends. 
Although eq 1 can be used with confidence for dense materials [20,21] some cares must be taken when electrokinetic measurements are performed with ion-permeable materials like most composite membranes which are made of a (dense or porous) skin layer on a porous support (which can be composed of one or several sublayers). If the membrane bodies are exposed to the hydrodynamic flow during electrokinetic experiments [22], a streaming current is likely to occur also within these porous structures. This has been recently demonstrated experimentally by Yaroshchuk and Luxbacher [17] with monolayer porous membranes. From an electrical point of view, the system behaves as a parallel circuit (Fig. 1) and the streaming current available from experiments $\left(I_{s}^{\text {tot }}\right)$ is therefore the sum of the current flowing through the channel $\left(I_{s}^{c h}\right)$ and that flowing through the porous body of membranes $\left(I_{s}^{m b}\right) . I_{s}^{m b}$ can be viewed as a parasite signal that must be quantified and subtracted from the experimental value of $I_{s}^{\text {tot }}$ to get the actual electrokinetic properties of membrane surfaces.

In this case eq 1 no longer holds and should be replaced by eq 2 [17]:

$$
I_{s}^{t o t}=I_{s}^{c h}+2 I_{s}^{m b}=-\left(\frac{W h_{c h} \varepsilon_{0} \varepsilon_{r} \Delta P}{\eta L} \zeta_{s u r f}+\frac{2 W h_{m b}^{e f f} \varepsilon_{0} \varepsilon_{r} \Delta P}{\eta L} \zeta_{m b}\right)
$$

where $\zeta_{\text {surf }}$ and $\zeta_{m b}$ are the zeta potentials of the membrane surface and the membrane body, respectively, and $h_{m b}^{\text {eff }}$ the effective height in which the streaming current flows through a single membrane body.

The effective height $h_{m b}^{\text {eff }}$ in eq 2 depends on the structural features of the porous substructure, i.e. its thickness, porosity and tortuosity. However, it should be pointed out that in the context of the present tangential electrokinetic measurements the membranes behave as a system of hydraulic resistances in parallel whereas a system of resistances in series is to be considered in standard filtration experiments. Because of the heterogeneous porous structure of the membranes, the values of both the porosity and the tortuosity may then differ somehow between these two different contexts. 
If the contribution of the membrane supports is not taken into account in the interpretation of electrokinetic data, the zeta potential of the membrane surfaces is then be overestimated (in absolute value) if the charge of both the surface and the porous body of membranes have the same sign whereas it is underestimated, or even opposite in sign with respect to the true zeta potential, if the surface and the porous structure carry opposite charges. Obviously, the error made in the determination of the surface zeta potential increases as the channel height is reduced due to the increasing fraction of the total streaming current flowing through the membrane bodies (the streaming current through the channel is proportional to the channel height while the streaming current through the membrane bodies is independent of the channel height).

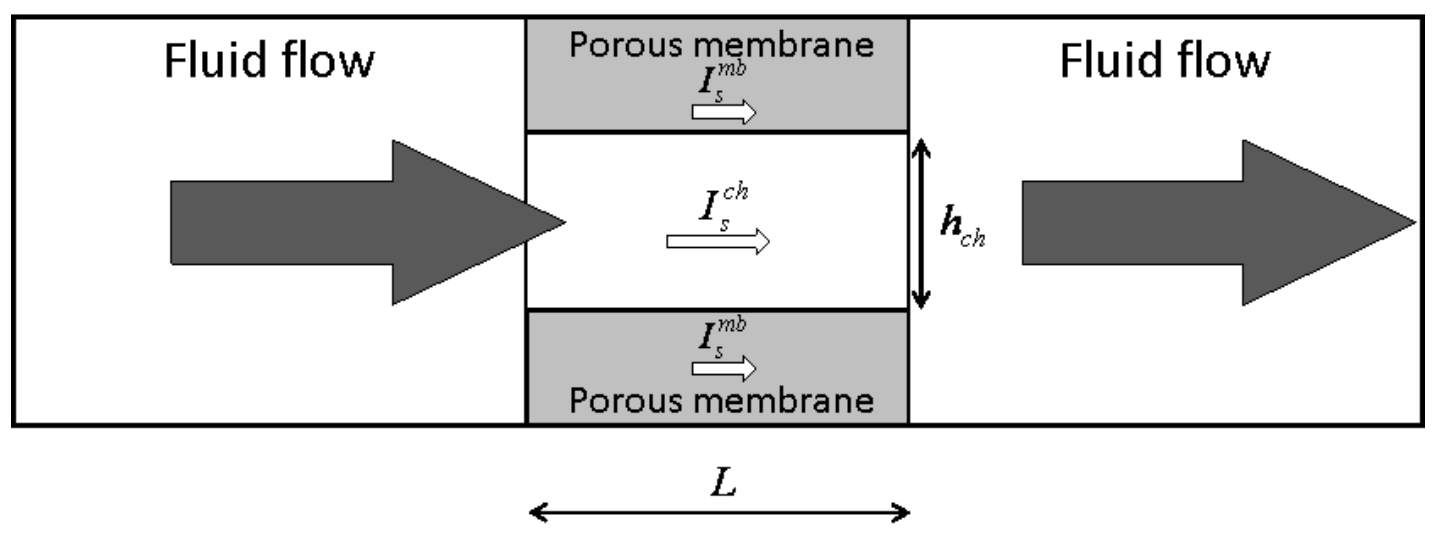

Fig. 1. Distribution of the streaming current during tangential electrokinetic experiments with membranes whose porous structures are exposed to the hydrodynamic fluid flow. $I_{s}^{c h}$ represents the streaming current flowing through the channel, $I_{s}^{m b}$ the streaming current flowing through the porous body of each membrane, $L$ is the channel length and $h_{c h}$ is the channel height. The streaming current measured experimentally $\left(I_{s}^{t o t}\right)$ is equal to $I_{s}^{t o t}=I_{s}^{c h}+2 I_{s}^{m b}$ (see eq 2). 
According to eq 2 the zeta potential of membrane surfaces can be deduced from the slope of $I_{s}^{\text {tot }}$ as function of $h_{c h}$ while the streaming current flowing through the two membrane porous bodies is given by the y-intercept (for dense materials the y-intercept would be zero). Although $I_{s}^{m b}$ can be considered as a parasite contribution to the experimental signal $\left(I_{s}^{\text {tot }}\right)$, it is worth noting that eq 2 shows that its knowledge makes possible the determination of the effective zeta potential of the porous structure of membrane $\left(\zeta_{m b}\right)$ provided that the value of $h_{m b}^{e f f}$ is known. It should be stressed that eq 2 was derived by assuming that both the channel height $\left(h_{c h}\right)$ and the pores of the membrane supports are large with respect to the Debye length. Although this condition is very likely fulfilled in the channel (in typical electrokinetic experiments $h_{c h}$ is several tens of micrometers while the Debye length does not exceed a few tens of nanometers). This may not be right inside the porous sublayers depending on their pore size, and $\zeta_{m b}$ should be viewed as an approximate value in the general case (for pore size close to the Debye length there is no analytical relation to link the streaming current to the zeta potential and numerical methods must be applied [23]).

The effective height in which the streaming current flows through a single membrane support $\left(h_{m b}^{e f f}\right)$ can be assessed from the measurement of the electric conductance of the system $\left(G_{c e l l}\right)$. If the surface conductance [24] in the channel is negligible (i.e. if the electric conductivity of the solution in the channel is identical to that of the bulk solution, $\left.\lambda_{0}\right), G_{c e l l}$ is related to $h_{m b}^{\text {eff }}$ as follows [17]:

$$
G_{c e l l}=\frac{W}{L}\left(h_{c h} \lambda_{0}+2 h_{m b}^{e f f} \lambda_{m b}\right)
$$

where $\lambda_{m b}$ is the electric conductivity of the electrolyte solution in the porous bodies of membranes.

If pores inside membrane bodies are not much larger than the Debye length $\lambda_{m b}$ can be much higher than $\lambda_{0}$. It is thus desirable to perform cell conductance measurements with a sufficiently high electrolyte 
concentration (e.g. $0.1 \mathrm{M}$ ) since the double-layer contribution to the overall conductivity is negligible at high ionic strength [25].

It should be stressed that the value of $h_{m b}^{\text {eff }}$ determined from electric conductance measurements and eq 3 is strictly equivalent to the effective thickness in which the streaming current flows through the membrane bodies (i.e. $h_{m b}^{\text {eff }}$ in eq 2 ) only in the case of sufficiently large pores. Indeed, although an electrolyte solution inside the porous structure contributes necessarily to the cell conductance, its contribution to the streaming current may well be negligible if pores are too narrow. In this case, the interpretation of the experimental streaming current would be straightforward since eq 1 is reliable but it is worth noting that the interpretation of experimental streaming potential would require taking into account the membrane body conductance according to procedures described in refs 13-15.

In other words, for small pores the value of $h_{m b}^{\text {eff }}$ to be used in eq 2 could be lower than the value determined from cell conductance measurements and eq 3. Unfortunately, it does not seem possible to estimate the value of $h_{m b}^{e f f}$ differently for composite membranes due to the complex structure of these materials.

\section{EXPERIMENTAL SECTION}

\section{Chemicals}

Sodium nitrite (Aldrich) and hypophosphorous acid (50wt\% in water; Aldrich) were used as received. (4-Aminobenzyl)-triphenylphosphonium bromide was obtained by the reduction of the commercial (4nitrobenzyl)-triphenylphosphonium bromide (Acros Organics) [26, 27].

Electrolyte solutions used in electrokinetic measurements were prepared from $\mathrm{KCl}$ (Fisher Scientific) and deionised water (resistivity: $18 \mathrm{M} \Omega \mathrm{cm}$ ) and a $0.1 \mathrm{M} \mathrm{HCl}$ solution (Fisher Scientific) was used to adjust their $\mathrm{pH}$. 


\section{Membranes}

HFK-131 ultrafiltration membranes (Koch Membrane Systems) were used in this work. Those membranes are composite materials made of a polyethersulfone (PES) layer (formed by phase inversion) on top of a non woven support in polyester. The whole membrane thickness is approximately $200 \mu \mathrm{m}$ while that of the PES asymmetric layer is about 60-70 $\mu \mathrm{m}$ (see Fig. 2). Membranes were first washed with deionised water (resistivity: $18 \mathrm{M} \Omega \mathrm{cm}$ ) and were sonicated $(2 \times 20$ minutes) in order to remove preservatives [28].

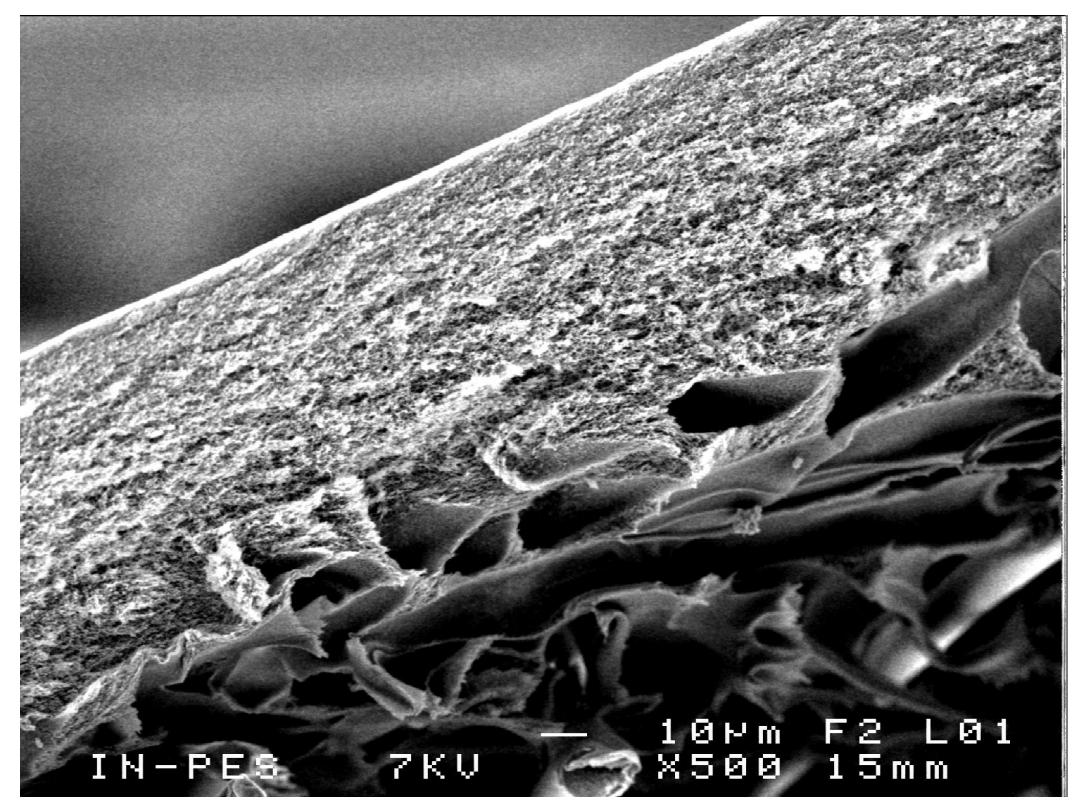

Fig. 2. SEM image of the cross-section of the HFK-131 composite membrane.

\section{Membrane functionalization}

Membranes were modified covalently by the method of chemical reduction of aryl diazonium salts [2932]. 4-Benzyltriphenylphosphonium diazonium (Fig. 3) was generated in situ in an acidic media (0.1 M $\mathrm{HCl}$ ) containing 50mM of (4-aminobenzyl)-triphenylphosphonium bromide and followed by addition of 
sodium nitrite at $100 \mathrm{mM}$. Immediately after that, $10 \mathrm{~mL}$ of hypophosphorous acid (chemical reductant) was added in the solution in order to reduce the in situ-generated aryl diazonium salt and form 4benzyltriphenylphosphonium radicals. Membranes were then dipped in the reactive solution and left to react overnight. After functionalization, the grafted membranes were rinsed with pure water and sonicated for 2 x 20 minutes in order to remove absorbed or loosely bound modifiers from their surfaces (like non covalently bound polyphenylene oligomers). The hydraulic permeability of membranes was found to decrease from 78 to $64 \mathrm{~L} \mathrm{~h}^{-1} \mathrm{~m}^{-2}$ after chemical modification, indicating a slight reduction of the average pore size [32].

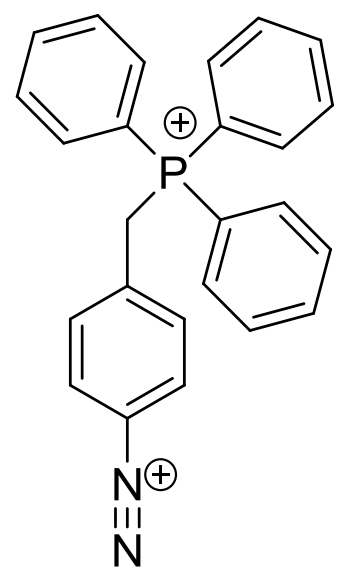

Fig. 3. Chemical structure of the in situ-generated 4-benzyltriphenylphosphonium diazonium.

\section{Electrokinetic measurements}

A SurPASS electrokinetic analyzer (Anton Paar $\mathrm{GmbH}$ ) was used to perform both streaming current and cell conductance measurements. All measurements were conducted with an adjustable-gap cell with which it is possible to vary the distance between the two membrane samples without dismounting the cell (thanks to micrometric screws). Rectangular membrane samples (dimensions: $L=20 \mathrm{~mm}$ and $W=$ $10 \mathrm{~mm}$ ) were fixed on sample holders using double-sided adhesive tape. To prevent any leakage 
between the membranes and the sample holders, membranes were firmly pressed against sample holders for 30-60 s, paying special attention to the edges exposed to the hydrodynamic flow.

Experiments were performed at $T=22 \pm 2^{\circ} \mathrm{C}$ with $500 \mathrm{~mL}$ of a $0.001 \mathrm{M} \mathrm{KCl}$ solution whose $\mathrm{pH}$ was adjusted at $5.5 \pm 0.1$ with a $0.1 \mathrm{M} \mathrm{HCl}$ solution. Prior to measurements, the solution was circulated through the channel for ca. 2 hours to allow for the sample equilibration. Streaming current was further measured by a pair of $\mathrm{Ag} / \mathrm{AgCl}$ electrodes (surface area: $10 \mathrm{~cm}^{2}$; this "large" surface area limits the electrode polarization during streaming current measurements), the equilibration process being monitored experimentally via the time dependence of the streaming current. After equilibration, the streaming current was measured and recorded for increasing pressure differences $(\Delta \mathrm{P})$ up to $300 \mathrm{mbar}$, the flow direction being changed periodically (which helps to limit electrode polarization). An illustration of typical streaming current measurements is shown in Fig. 4. Measurements were repeated three times so as to evaluate experimental errors associated with the determination of the streaming current. 


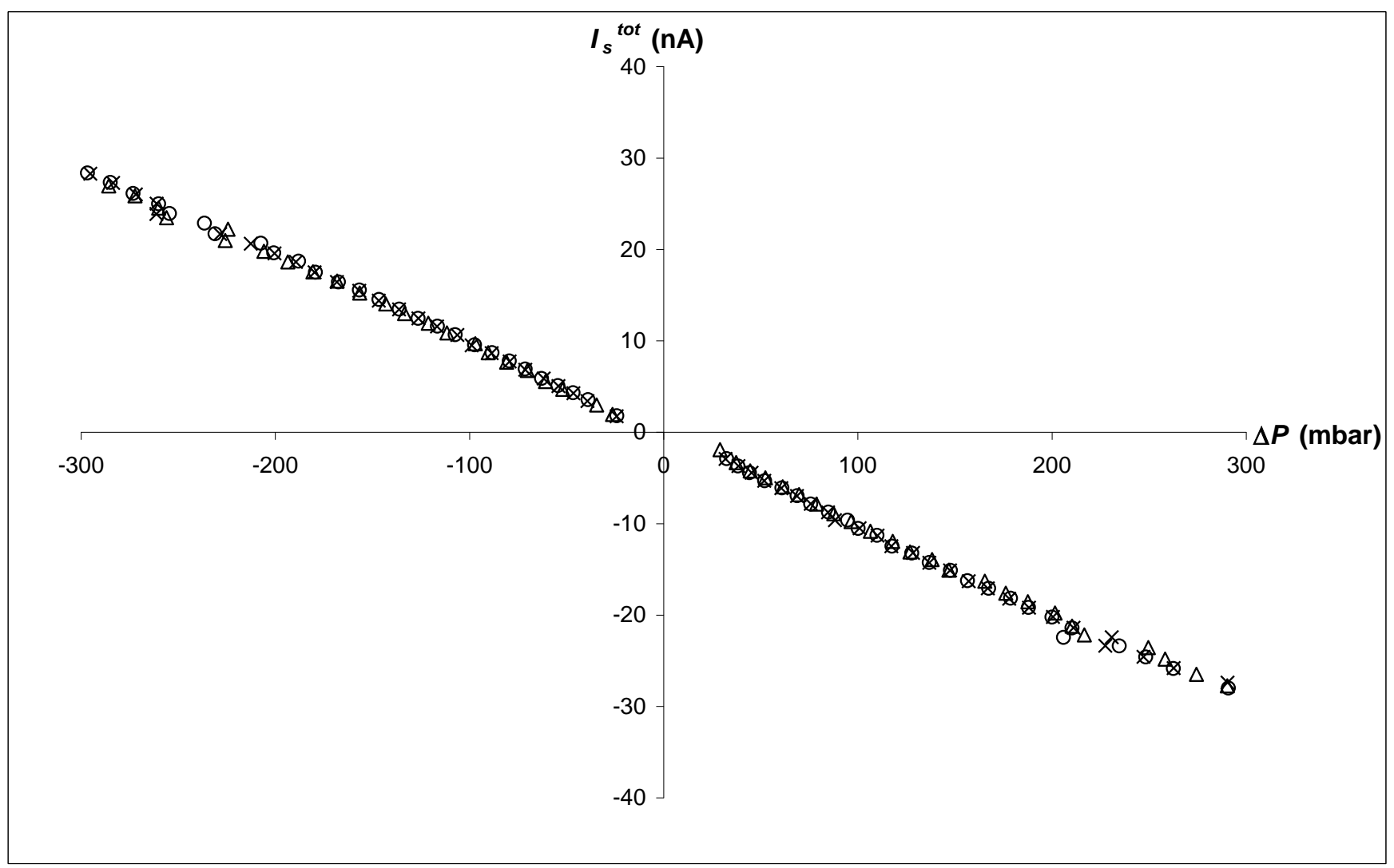

Fig. 4. Example of streaming current measurements performed in alternating flow directions (unmodified HFK-131 membranes; channel height: $103 \pm 2 \mu \mathrm{m}$ ).

The channel height $\left(h_{c h}\right)$ was varied between $\sim 40$ and $\sim 110 \mu \mathrm{m}$ by means of micrometric screws and its value was determined from volume flow rate $\left(Q_{V}\right)$ measurements performed at various $\Delta \mathrm{P}$ by means of the Hagen-Poiseuille relation which reads as follows for parallelepipedic channels (considering that the contribution of porous structures to $Q_{V}$ is negligible [17] and neglecting edge phenomena):

$$
h_{c h}=\sqrt[3]{\frac{12 \eta L Q_{V}}{W \Delta P}}
$$

The experimental error associated with the determination of $h_{c h}$ was found to be $\pm 2 \mu \mathrm{m}$. 
The Surpass electrokinetic analyzer also allows the measurement of the electrical conductance of the test cell $\left(G_{\text {cell }}\right)$ in a.c. mode at $160 \mathrm{~Hz}$ (the same electrodes are used as in electrokinetic measurements). The reliability of measured conductances was checked by performing additional streaming potential measurements (the Surpass instrument allows measurements of both the streaming potential and the streaming current with the same pair of electrodes thanks to automatic commutation of the external electrical circuit) and by computing the (direct current) conductance from the streaming current $\left(I_{s}^{t o t}\right)$ to streaming potential $\left(\Delta V_{s}\right)$ ratio. A very good agreement was obtained for all membranes as illustrated in Fig. 5 for unmodified HFK-131 membrane samples. Although conductances measured with alternating or direct currents may be not identical in heterogeneous media [17], the very good agreement obtained between these three independent measurements (conductance, streaming current and streaming potential) gives evidence of the reliability of both electric and electrokinetic measurements performed in this work. 


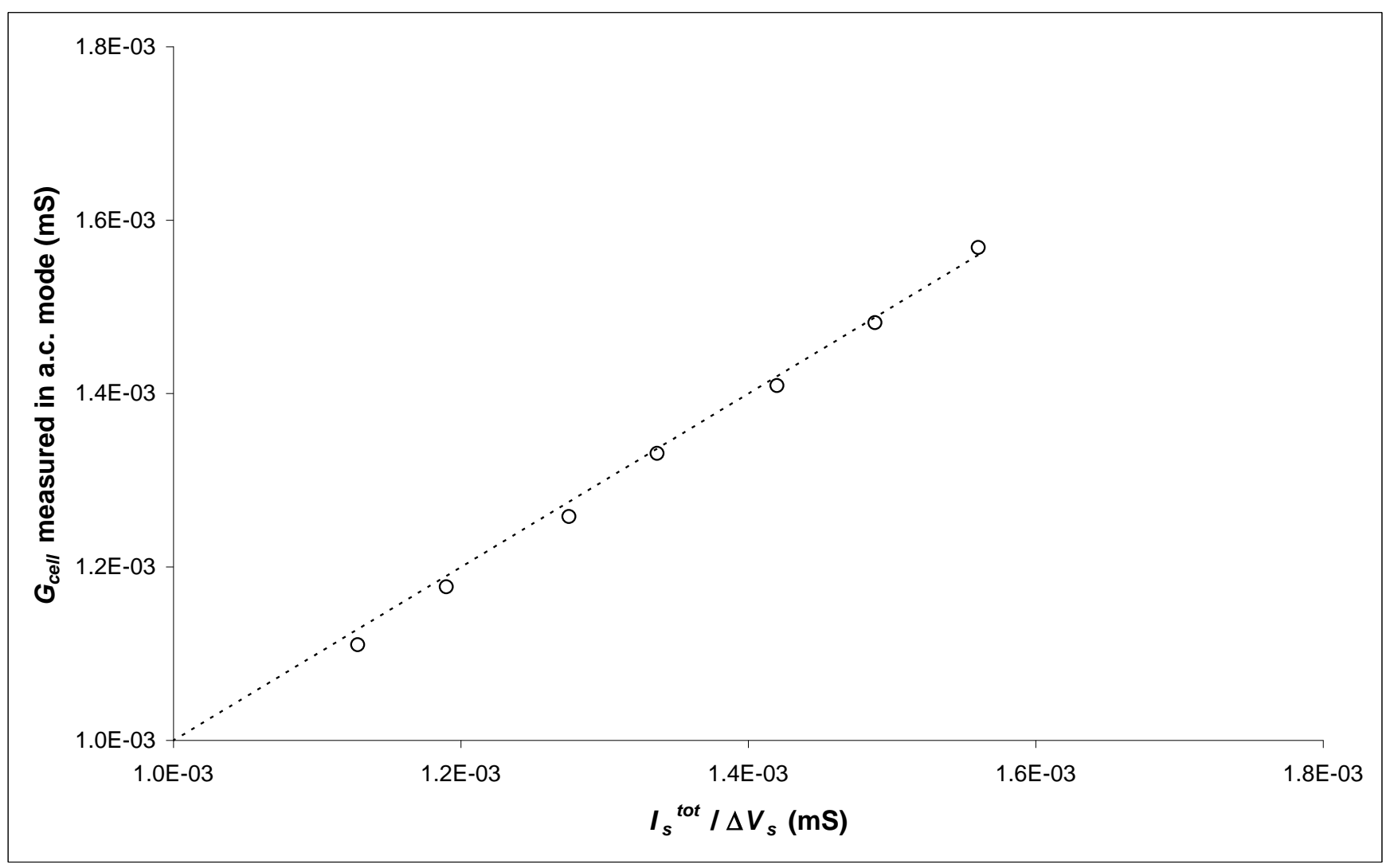

Fig. 5. Illustration of the very good agreement between the cell conductance measured in a.c. mode at $160 \mathrm{~Hz}$ and the ratio between the streaming current and the streaming potential (which corresponds to the conductance measurable with direct current). The dotted line shows the exact equality between both quantities and the symbols correspond to experimental results obtained for the same membrane samples as in Fig. 4 with channel heights ranging from 52 to $114 \pm 2 \mu \mathrm{m}$.

The determination of the cell conductance is useful to get insight into the electrokinetic properties of the membrane porous bodies [17]. Indeed, according to eq 3 the variation of the cell electrical conductance with respect to the channel height allows determining $h_{m b}^{\text {eff }}$. Depending on the pore size and the zeta potential of membrane bodies, the electric conductivity inside the membrane can be higher than the bulk conductivity. This effect is suppressed at high salt concentrations [25]. Therefore, we performed electrical resistance measurements for two different $\mathrm{KCl}$ concentrations, 0.001 and $0.1 \mathrm{M}$. Cell conductance measurements were repeated three times. 
To account for the variability of membrane properties coming from different batches, functionalization and electrokinetic characterization were performed with membranes samples from two different batches (of course the comparison between unmodified and modified membranes was performed with membrane samples coming from the same batch).

\section{RESULTS AND DISCUSSION}

Fig. 6 shows the experimental streaming current coefficient measured with HFK-131 membranes (coming from two different batches) at various channel heights $\left(h_{c h}\right)$ before and after functionalization by positively charged 4-benzyltriphenylphosphonium groups (hereafter referred to as $\mathrm{P}+$ ). 


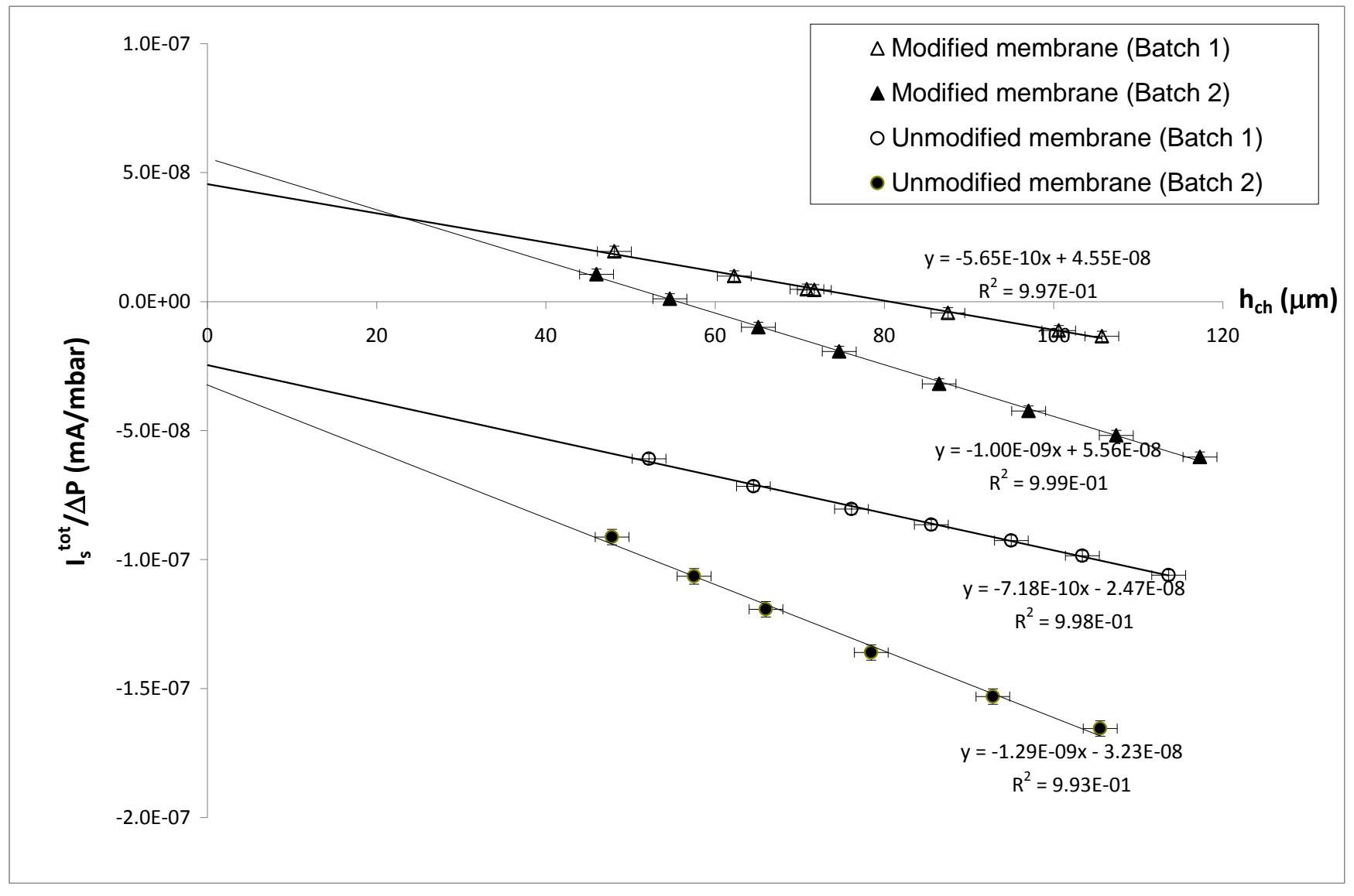

Fig. 6. Streaming current coefficient $\left(I_{s}^{\text {tot }} / \Delta P\right)$ versus channel height $\left(h_{c h}\right)$ measured with HFK-131 PES membranes before and after functionalization by 4-benzyltriphenylphosphonium groups $(\mathrm{P}+)$. Experiments were performed in a $0.001 \mathrm{M} \mathrm{KCl}$ solution at $\mathrm{pH} 5.50 \pm 0.05$.

As expected, the total streaming current measured through the cell varies linearly with the channel height. However, for all membranes the lines do not pass through the origin. This gives evidence that an additional streaming current flows through the porous structures of membranes, the value of which is obtained from extrapolation at zero channel height (y-intercept in Fig. 6). These findings confirm the recent results obtained by Yaroshchuk and Luxbacher who demonstrated the occurrence of a streaming current through the porous body of homogenous membranes during tangential electrokinetic experiments [17]. 
A rather important variability of the electrokinetic properties can be noticed between membrane samples coming from the two different batches. The interpretation of the electrokinetic properties of modified membranes is then to be made by comparison with the electrokinetic properties of an unmodified membrane coming from the same batch. Whatever the membrane batch, the slopes of the lines $I_{s}^{t o t} / \Delta P=f\left(h_{c h}\right)$ were found to decrease (in absolute value) after membrane functionalization meaning that the membrane surfaces were modified by $\mathrm{P}+$ groups. The surface zeta potential $\left(\zeta_{\text {surf }}\right)$ was deduced straightforwardly for the different membranes from eq 2 and the slopes obtained in Fig. 6. The results are shown in Fig. 7 which indicates that only a moderate amount of positively charged groups were actually grafted onto the external surface of HFK-131 membranes since $\zeta_{\text {surf }}$ of the modified membranes was only slightly reduced with respect to that of the unmodified membranes.

A striking result in Fig. 6 is the sign reversal of the streaming current coefficient that was obtained with the modified membranes below some value of the channel height. This is a clear indication that the porous structure and the external surface of modified membranes carry an electrical charge of opposite sign (whatever the membrane batch). In other words, the membrane bodies are positively charged after functionalization while the external surface remains negatively charged. 


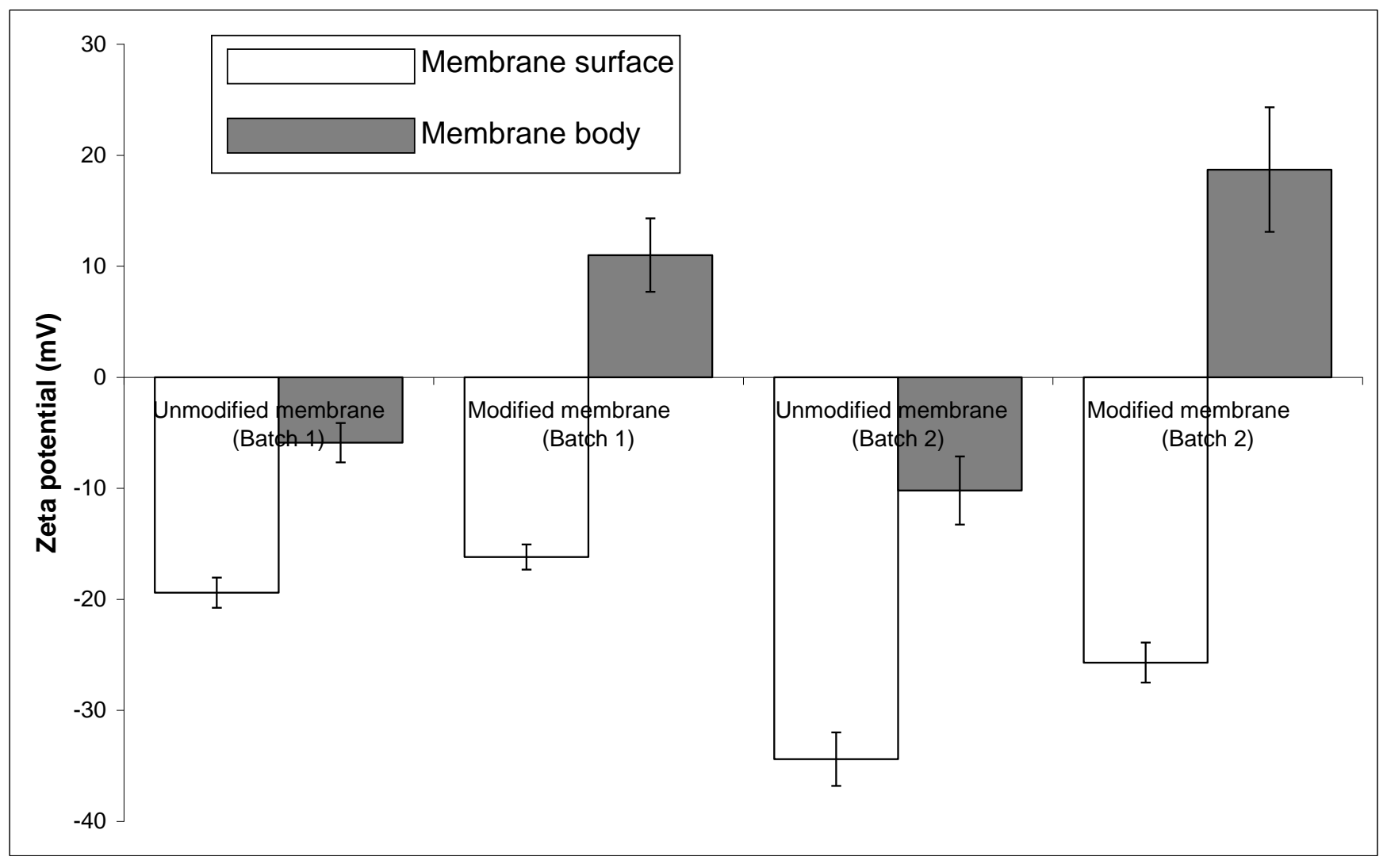

Fig. 7. Zeta potential of the surface and porous body of HFK-131 PES membranes before and after functionalization by 4-benzyltriphenylphosphonium groups $(\mathrm{P}+)$. Experiments were performed in a $0.001 \mathrm{M} \mathrm{KCl}$ solution at $\mathrm{pH} 5.50 \pm 0.05$.

According to eq 2, the zeta potential of the membrane bodies can be obtained from the y-intercept of lines shown in Fig. 6 (which corresponds to the streaming current flowing through the two porous structures during electrokinetic experiments) provided that the effective thickness in which the current flows through a single membrane body $\left(h_{m b}^{\text {eff }}\right)$ is known. As shown by eq $3, h_{m b}^{\text {eff }}$ can be estimated by extrapolation from a series of measurements of cell conductance $\left(G_{\text {cell }}\right)$ performed at various channel heights. However, it must be stressed that eq 3 assumes that the surface conductance phenomenon in the channel is negligible (i.e. the electric conductivity of the solution in the channel is identical to that of the bulk solution). This seems to be a relevant approximation in our case since (i) the channel heights under consideration are much larger than the Debye length of the system (around $10 \mathrm{~nm}$ for a millimolar 
solution of mono-monovalent electrolyte), and (ii) the surface zeta potentials of HFK-131 membranes (both unmodified and modified by $\mathrm{P}+$ ) are rather low. The determination of $h_{m b}^{\text {eff }}$ from eq 3 also requires the knowledge of the actual conductivity of the electrolyte solution that fills the porous bodies of membranes $\left(\lambda_{m b}\right)$. Unfortunately, in most cases $\lambda_{m b}$ cannot be determined experimentally due to the very complex structure of composite membranes. Nevertheless, $\lambda_{m b}$ can be considered to be equal to the bulk-solution conductivity $\left(\lambda_{0}\right)$ if pores are large enough with respect to the Debye length and the pore walls are not too highly charged. In order to check the relevance of the approximation $\lambda_{m b} \approx \lambda_{0}$ for the membranes under consideration, we performed cell conductance measurements versus channel height with 0.001 and $0.1 \mathrm{M} \mathrm{KCl}$ solutions. Indeed, the contribution of electrical double-layers to the overall conductivity inside the membrane bodies is negligible in a $0.1 \mathrm{M}$ electrolyte solution and the equality between $\lambda_{0}$ and $\lambda_{m b}$ is therefore expected [33].

Results obtained with the different membrane samples in a $0.001 \mathrm{M} \mathrm{KCl}$ solution are shown in Fig. 8. The cell conductance was found to vary linearly with $h_{c h}$ for all membranes as expected from eq 3 . Similar linear relations were obtained with a $0.1 \mathrm{M} \mathrm{KCl} \mathrm{M}$ solution (results not shown). Assuming $\lambda_{m b} \approx \lambda_{0}$, the y-intercept was used to determine $h_{m b}^{\text {eff }}$ using eq 3 . The values obtained for the different membranes are collected in Table 1. No significant discrepancy was observed between values determined in $0.001 \mathrm{M}$ and $0.1 \mathrm{M} \mathrm{KCl}$ solutions. This gives evidence that the approximation $\lambda_{m b} \approx \lambda_{0}$ is reliable for these membranes (at least for electrolyte concentrations down to $0.001 \mathrm{M}$ ). According to eq 3 and considering the dimensions of the membrane samples given in section 3, the slopes of lines shown in Fig. 8 should be equal to half the bulk solution conductivity. Direct measurements of bulk solution conductivities were also performed and values were found to differ from results inferred from Fig. 8 by less than $5 \%$ for all solutions. The slightly different slopes observed in Fig. 8 (corresponding to bulk conductivities between 129 and $151 \mu \mathrm{S} \mathrm{cm}^{-1}$ ) are due to the fact that experiments were carried out at different temperatures ranging from $20-24^{\circ} \mathrm{C}$ (see section 3 ). 


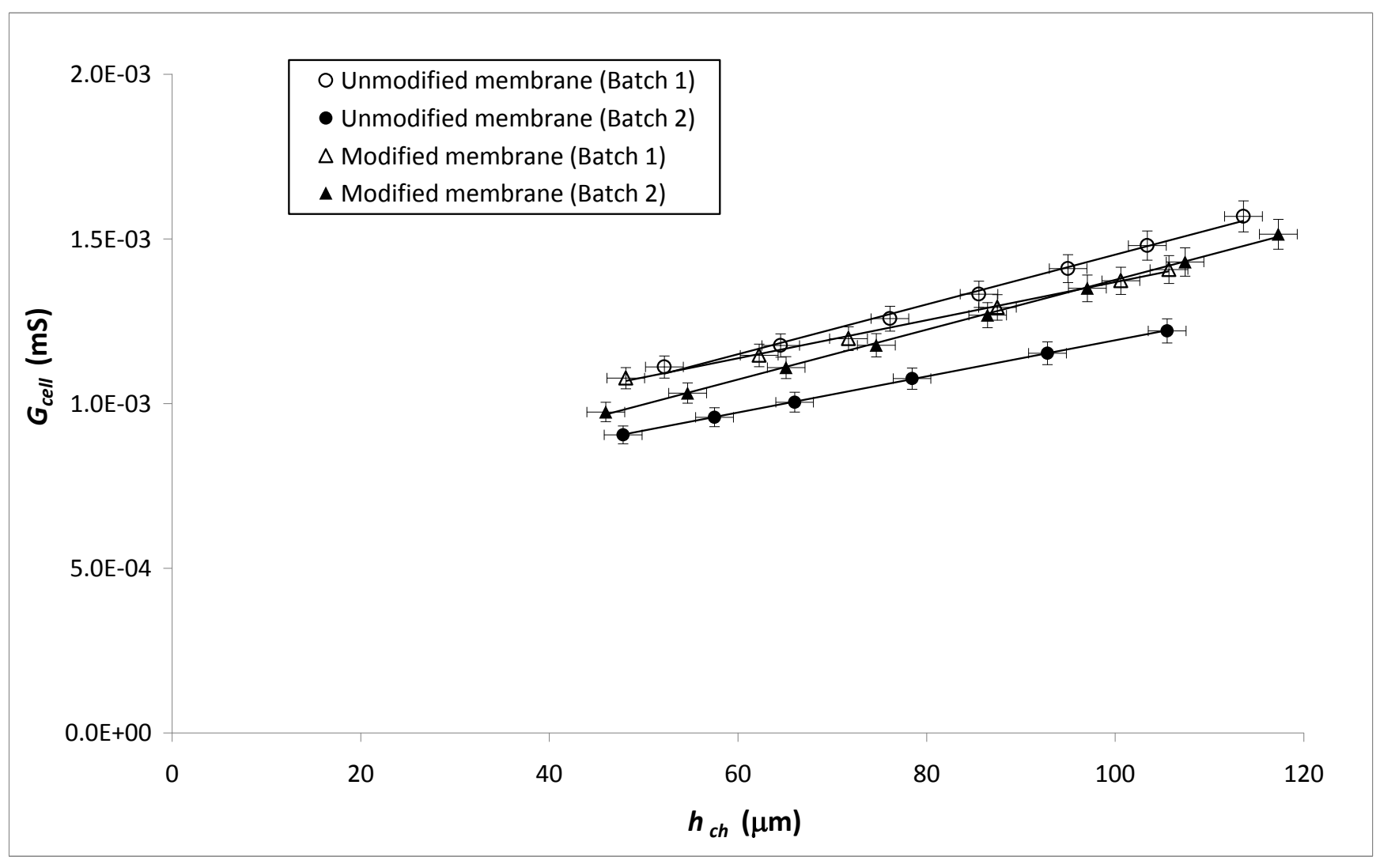

Fig. 8. Electric conductance $\left(G_{c e l l}\right)$ versus channel height $\left(h_{c h}\right)$ measured with HFK-131 PES membranes before and after functionalization by 4-benzyltriphenylphosphonium groups $(\mathrm{P}+)$. Experiments were performed in a $0.001 \mathrm{M} \mathrm{KCl}$ solution at $\mathrm{pH} 5.50 \pm 0.05$.

According to the experimental errors associated with the determination of $h_{m b}^{\text {eff }}$ (see Table 1) it seems that the functionalization process does not modify significantly the value of $h_{m b}^{e f f}$. We also suspect that $h_{m b}^{e f f}$ can be somehow dependent on the way the polymer membranes are fixed onto the sample holders. As mentioned in the experimental section, membranes were fixed on sample holders using double-sided adhesive tape and firmly pressed against sample holders to avoid any possible leakage between the membranes and the sample holders during electrokinetic experiments. During this step, it might be possible that the adhesive tape clogs a small part of the membrane support. If so, then the actual part of a 
membrane that is effectively exposed to the hydrodynamic flow during experiments does not correspond exactly to the total membrane thickness and could be slightly different from a sample to another. It is therefore recommended to always perform the conductance measurements described above when new membrane samples are inserted in the test cell. It is worth noting that the values obtained for $h_{m b}^{e f f}$ are physically relevant since the total thickness (polyester support + PES active layer) of the HFK-131 membrane is around $200 \mu \mathrm{m}$.

\begin{tabular}{|c|c|c|}
\hline & $\begin{array}{c}h_{m b}^{e f f}(\mu \mathrm{m}) \text { determined in } \\
\mathrm{KCl} 0.001 \mathrm{M}\end{array}$ & $\begin{array}{c}h_{m b}^{e f f}(\mu \mathrm{m}) \text { determined in } \\
\mathrm{KCl} 0.1 \mathrm{M}\end{array}$ \\
\hline Unmodified membrane (Batch 1) & $52 \pm 6$ & $46 \pm 7$ \\
\hline Modified membrane (Batch 1) & $63 \pm 5$ & $62 \pm 8$ \\
\hline Unmodified membrane (Batch 2) & $58 \pm 10$ & $60 \pm 5$ \\
\hline Modified membrane (Batch 2) & $42 \pm 8$ & $44 \pm 5$ \\
\hline
\end{tabular}

Table 1. Values of $h_{m b}^{\text {eff }}$ determined from cell conductance measurements in 0.001 and $0.1 \mathrm{KCl}$ solutions at $\mathrm{pH}=5.50 \pm 0.05$.

Knowing $h_{m b}^{e f f}$, the effective zeta potentials of the different membrane bodies $\left(\zeta_{m b}\right)$ were determined from eq 2 and the streaming current coefficients extrapolated at zero channel height (Fig. 6). Results obtained with both unmodified and modified HFK-131 membranes are depicted in Fig. 7. The porous body of unmodified membranes is negatively charged. On the other hand, the zeta potential of the porous body of modified membranes was found to be positive. This gives evidence for the occurrence of grafting inside the porous structure of membrane. From the comparison between the different zeta potentials shown in Fig. 7 it can be concluded that the functionalization of the polyethersulfone 
membrane is more important inside the membrane-support pores that on its external surface. It should be stressed also that the determination of the zeta potential of membrane surfaces from the slopes of the lines $I_{s}^{t o t} / \Delta P=f\left(h_{c h}\right)$ is much more accurate than that of the zeta potential of membrane bodies (see Fig. 7) which is obtained from the y-intercepts of lines $I_{s}^{t o t} / \Delta P=f\left(h_{c h}\right)$ and $G_{c e l l}=f\left(h_{c h}\right)$.

\section{CONCLUSION}

It was shown that a fraction of the streaming current measured in tangential electrokinetic experiments performed with composite polymer membranes may flow through the underlying porous structure of membranes. The relative contribution of this parasite signal to the overall streaming current available experimentally depends on both the structural and electrical features of membrane bodies (i.e. their underlying porous structures including intermediate layer(s) and / or support layer). The experimental procedure proposed by Yaroshchuk and Luxbacher (based on tangential streaming current and electrical conductance measurements) [17] was used to produce a correct interpretation of experimental data. This made possible to separate the contributions of the external surface and that of the porous structure for an ultrafiltration composite membrane. The method was shown to bring useful information about the efficiency of the functionalization of this membrane by chemical reduction of an aryldiazonium salt bearing a positively charged group. It revealed some interesting details concerning the pattern of the membrane modification, notably that the functionalization primarily occurs in the underlying porous structure rather than on its external surface. The advanced electrokinetic characterization performed in this work could also provide significant insights into important issues in membrane science including membrane ageing and fouling. 


\section{APPENDIX - LIST OF SYMBOLS}

\section{Roman letters}

F: $\quad$ Faraday constant $\left(96485 \mathrm{C} \mathrm{mol}^{-1}\right)$

$G_{\text {cell }}: \quad$ Electric conductance of the cell $\left(\Omega^{-1}\right)$

$h_{c h}: \quad$ Channel height $(\mathrm{m})$

$h_{m b}^{e f f}$ : Effective height in which the streaming current flows through the membrane porous body $(\mathrm{m})$

I: $\quad$ Ionic strength $\left(\mathrm{mol} \mathrm{L}^{-1}\right)$

$I_{s}: \quad$ Streaming current $(\mathrm{A})$

$I_{s}^{c h}$ : Streaming current flowing through the channel (A)

$I_{s}^{m b}$ : Streaming current flowing through the membrane porous body (A)

$I_{s}^{\text {tot }}$ : Total streaming current flowing through the cell (A)

$L: \quad$ Channel length $(\mathrm{m})$

$Q_{V}: \quad$ Volume flow rate $\left(\mathrm{m}^{3} \mathrm{~s}^{-1}\right)$

$R: \quad$ Ideal gas constant $\left(8.314 \mathrm{~J} \mathrm{~mol}^{-1} \mathrm{~K}^{-1}\right)$

T: $\quad$ Temperature $(\mathrm{K})$

W: $\quad$ Channel width (m) 


\section{Greek letters}

$\Delta P: \quad$ Hydrostatic pressure difference between channel ends $\left(\mathrm{N} \mathrm{m}^{-2}\right)$

$\Delta V_{s}: \quad$ Streaming potential (V)

$\varepsilon_{0}: \quad$ Vacuum permittivity $\left(8.854 \times 10^{-12} \mathrm{~F} \mathrm{~m}^{-1}\right)$

$\varepsilon_{r}: \quad$ Dielectric constant of the solution (-)

$\eta: \quad$ Viscosity of the solution $\left(\mathrm{kg} \mathrm{m}^{-1} \mathrm{~s}^{-1}\right)$

$\kappa^{-1}: \quad$ Debye length $(\mathrm{m})$

$\lambda_{0}: \quad$ Electric conductivity of bulk solution $\left(\Omega^{-1} \mathrm{~m}^{-1}\right)$

$\lambda_{m b}: \quad$ Electric conductivity of the solution in the membrane porous body $\left(\Omega^{-1} \mathrm{~m}^{-1}\right)$

$\zeta: \quad$ Zeta potential $(\mathrm{V})$

$\zeta_{m b}: \quad$ Effective zeta potential of the membrane porous body (V)

$\zeta_{\text {surf }}$ : Zeta potential of the membrane surface $(\mathrm{V})$

\section{ACKNOWLEDGMENT}

This research work was supported by funding from the "Agence Nationale de la Recherche" through MUTINA project (ANR 2011 BS09 002) and by the European Union's Seventh Framework Programme FP7/2007-2013 under Grant Agreement No. 226532. 


\section{REFERENCES}

[1] C. Labbez, P. Fievet, F. Thomas, A. Szymczyk, A Vidonne, A. Foissy, J. Pagetti, Evaluation of the DSPM model on a titania membrane: measurements of charged and uncharged solute retention, electrokinetic charge, pore size and water permeability. J. Colloid Interface Sci. 262 (2003) 200-211.

[2] M.G.A. Khedr, S.M. Abdel Halleem, A. Bakara, Selective behavior of hyperfiltration cellulose acetate membranes. J. Electroanal. Chem. 184 (1985) 161-169.

[3] M. Nyström, M. Lindström, E. Matthiasson, Streaming potential as a tool in the characterisation of UF membranes. Coll. Surf. A 36 (1989) 297-312.

[4] M. Nyström, A. Pihlajamäki, N. Ehsani, Characterization of ultrafiltration membranes by simultaneous streaming potential and flux measurements. J. Membrane. Sci. 87 (1994) 245-256.

[5] I.H. Huisman, G. Trägardh, C. Trägardh, A. Pihlajamäki, Determining the zeta-potential of ceramic microfiltration membranes using the electroviscous effect. J. Membrane. Sci. 147 (1998) 187-194.

[6] J. Benavente, G. Jonsson, Effects of adsorbed protein on the hydraulic permeability, membrane and streaming potential Coll. Surf. A 138 (1998) 255-264.

[7] I.H. Huisman, P. Pradanos, A. Hernandez, Electrokinetic characterisation of ultrafiltration membranes by streaming potential, electroviscous effect, and salt retention. J. Membrane. Sci. 178 (2000) 55-64.

[8] A. Szymczyk, P. Fievet, J.C. Reggiani, J. Pagetti Determination of the filtering layer electrokinetic properties of a multilayer ceramic membrane. Desalination 116 (1998) 81-88.

[9] A. Szymczyk, C. Labbez, P. Fievet, B. Aoubiza, C. Simon, Streaming potential through multilayer membranes. AIChE J. 47 (2001) 2349-2358.

[10] A.E. Yaroshchuk, Y.P. Boiko A.L. Makovetskiy, Filtration potential across membranes containing selective layers. Langmuir 18 (2002) 5154-5162.

[11] A. Szymczyk, M. Sbaï, P. Fievet, Analysis of the pressure-induced potential arising through composite membranes with selective surface layers. Langmuir 21 (2005) 1818-1826.

[12] D. Möckel, E. Staude, M. Dal-Cin, K. Darcovich, M. Guiver, Tangential flow streaming potential measurements: hydrodynamic cell characterization and zeta potentials of carboxylated polysulfone membranes. J. Membrane. Sci. 145 (1998) 211-222.

[13] A.E. Yaroshchuk, V. Ribitsch, Role of channel wall conductance in the determination of $\zeta$ potential from electrokinetic measurements. Langmuir 18 (2002) 2036-2038. 
[14] P. Fievet, M. Sbaï, A. Szymczyk, A. Vidonne, Determining the $\zeta$-potential of plane membranes from tangential streaming potential measurements: effect of the membrane body conductance. J. Membrane Sci. 226 (2003) 227-236.

[15] M. Sbaï, A. Szymczyk, P. Fievet, A. Sorin, A. Vidonne, S. Pellet-Rostaing, A. Favre-Réguillon, M. Lemaire, Influence of the membrane pore conductance on tangential streaming potential. Langmuir 19 (2003) 8867-8871.

[16] T. Luxbacher, Electrokinetic characterization of flat sheet membranes by streaming current measurement. Desalination 199 (2006) 376-377.

[17] A.E. Yaroshchuk, T. Luxbacher, Interpretation of Electrokinetic Measurements with Porous Films: Role of Electric Conductance and Streaming Current within Porous Structure. Langmuir 26 (2010) 10882-10889.

[18] F. Lu, T.Y. How, Y. Kwok, An improved method for determining zeta potential and pore conductivity of porous materials. J. Colloid Interface Sci. 299 (2006) 972-976.

[19] The Debye length $\left(\kappa^{-1}\right)$ is defined as $\kappa^{-1}=\sqrt{\varepsilon_{0} \varepsilon_{r} R T /\left(2 F^{2} I\right)}$ where $\varepsilon_{0}$ is the vacuum permittivity, $\varepsilon_{\mathrm{r}}$ the relative dielectric constant of the solution, $R$ the ideal gas constant, $T$ the temperature, $F$ the Faraday constant and $I$ the ionic strength of the solution.

[20] C. Werner, H. Körber, R. Zimmermann, S. Dukhin, H.J. Jacobasch, Extended Electrokinetic Characterization of Flat Solid Surfaces. J. Colloid Interface Sci. 208 (1998) 329-346.

[21] R. Zimmermann, N. Rein, C. Werner, Water ion adsorption dominates charging at nonpolar polymer surfaces in multivalent electrolytes. Phys. Chem. Chem. Phys. 11 (2009) 4360-4364.

[22] Note that this seems to be the case for measuring cells currently available with commercial electrokinetic analyzers.

[23] A. Szymczyk, B. Aoubiza, P. Fievet, J. Pagetti, J. Electrokinetic phenomena in homogeneous cylindrical pores. J. Colloid Interface Sci. 216 (1999) 285-296.

[24] J. Lyklema, Fundamentals of Interface and Colloid Science, Vol. II, Academic Press, 1995.

[25] A. Szymczyk, P. Fievet, B. Aoubiza, C. Simon, J. Pagetti, An application of the space charge model to the electrolyte conductivity inside a charged microporous membrane. J. Membrane Sci. 161 (1999) 275-285.

[26] F.D. Bellamy, K. Ou, Selective reduction of aromatic nitro compounds with stannous chloride in non acidic and non aqueous medium. Tetrahedron Lett. 25 (1984) 839-842. 
[27] M. Picot, L. Lapinsonnière, M. Rothballer, F. Barrière, Graphite anode surface modification with controlled reduction of specific aryl diazonium salts for improved microbial fuel cells power output. Biosens. Bioelectron. 28 (2011) 181-188.

[28] S.X. Liu, J.T. Kim, S. Kim, Effect of polymer surface modification on polymer-protein interaction via hydrophilic polymer grafting. J. Food. Sci. 73 (2008) 143-150.

[29] J. Lyskawa, D. Bélanger, Direct modification of a gold electrode with aminophenyl groups by electrochemical reduction of in situ generated aminophenyl monodiazonium cations. Chem. Mater. 18 (2006) 4755-4763.

[30] S. Baranton, D. Bélanger, Electrochemical derivatization of carbon surface by reduction of in situ generated diazonium cations. J. Phys. Chem. B. 109 (2005) 24401-24410.

[31] F. Barrière, A.J. Downard, Covalent Modification of Carbon Surfaces by Non-Electrochemical Methods J. Sol. State Electrochem. 12 (2008) 1231-1244.

[32] M. Picot, R. Rodulfo, I. Nicolas, A. Szymczyk, F. Barrière, M. Rabiller-Baudry, A versatile route to modify polyethersulfone membranes by chemical reduction of aryldiazonium salts. J. Membrane Sci. 417-418 (2012) 131-136.

[33] M. Sbaï, P. Fievet, A. Szymczyk, B. Aoubiza, A. Vidonne, A. Foissy, Streaming potential, electroviscous effect, pore conductivity and membrane potential for the determination of the surface potential of a ceramic ultrafiltration membrane. J. Membrane Sci. 215 (2003) 1-9. 